\title{
Presence of Anterior Temporal Artery Associates with Good Outcome in Acute Atherosclerotic
}

\section{M1-Middle Cerebral Artery Occlusion}

Dezhi Liu, MD1 ${ }^{\#}$

Yongkun Li, MD, PhD2 ${ }^{\#}$

Zhaorong Shi, MD ${ }^{1}$

Stephen M. Davis, MD, FRACP $P^{3,4}$

Ka Sing Wong, MD ${ }^{5}$

Thomas W. Leung, MD ${ }^{5}$

Bernard Yan, $\mathrm{MD}^{3,4}$

Yunyun Xiong, MD, $\mathrm{PhD}$

Wen Sun, $\mathrm{MD}^{1}$

Gelin $\mathrm{Xu}, \mathrm{MD}, \mathrm{PhD}^{1}$

Renliang Zhang, $\mathrm{MD}, \mathrm{PhD}^{1}$

Xinfeng Liu, $\mathrm{MD}, \mathrm{PhD}^{1 *}$

${ }^{1}$ Department of Neurology, Jinling Hospital, Nanjing University School of Medicine, 305 East Zhongshan Road, Nanjing, 210002, Jiangsu Province, PR China

e-mail: xfliu2@vip.163.com

${ }^{2}$ Department of Neurology, Fujian Provincial Hospital, Provincial Clinical Department of Fujian Medical University, Fuzhou, China

${ }^{3}$ Melbourne Brain Centre, Royal Melbourne Hospital, Melbourne, Australia

${ }^{4}$ Department of Medicine, University of Melbourne, Melbourne, Australia 
${ }^{5}$ Department of Medicine and Therapeutics, Division of Neurology, Prince of Wales Hospital, Shatin, Hong Kong

"Dezhi Liu and Yongkun Li contributed equally to this work

*Corresponding author:

Xinfeng Liu

Tel: +86 2584801861

Fax: +86 2584805169 


\begin{abstract}
Introduction We aim to investigate the correlation between presence of Anterior temporal artery (ATA), the first major branch of middle cerebral artery (MCA), on conventional angiography and clinical outcome in patients with acute atherosclerotic M1-MCA occlusion.
\end{abstract}

Methods Consecutive patients with acute atherosclerotic M1-MCA occlusion from Nanjing Stroke Registry Program (NSRP) between January 2007 and December 2012 were included in this study. All patients underwent MRI followed by conventional angiography. From their data, we analyzed baseline characteristics, infarction patterns, DWI-ASPECTS and collateral circulation. The correlation of ATA presence and good clinical outcome, modified Rankin Scale (mRS) score $\leq 2$, at 3 months was also calculated.

Results In 98 patients meeting entry criteria, the presence of ATA was found in 44 patients. Patients with ATA present were found to have less hypertension $(p=0.042)$, lower baseline National Institutes of Health Stroke Scale (NIHSS) $(p=0.043)$, more small infarcts in perforating artery territory $(p=0.013)$ and a higher number of DWI-ASPECTS $\geq 7$ ( $p=0.034)$. Binary logistic regression analysis showed an adjusted odds ratio of 4.45 for a good outcome in patients with ATA presence (95\%CI: 1.52 to $13.03, p=0.007)$.

Conclusions The presence of ATA can be used as a predictor of good outcome in patients with acute atherosclerotic M1-MCA occlusion.

\title{
Keywords
}

stroke; middle cerebral artery occlusion; collateral status; lesion pattern

\section{Introduction}

There is an increasing amount of evidence supporting the notion that early identification of patients at risk for bad outcome is of great importance for acute atherosclerotic M1-middle cerebral artery (MCA) occlusion therapeutic decisions. [1] Several factors have been identified as important predictors of these patient outcomes, including stroke severity, patients' age, and infarct size. [2] However, these factors 
suffer from a lack of definitive predictive power.[3] Thus, there may be other valuable factors involved in the prediction of good outcome for these patients.

For acute atherosclerotic M1-MCA occlusion patients, collateral circulation may play an important role in influencing recovery through sustaining downstream perfusion distal to the occlusion or through enhancing embolic washout in distal arteries. [4] In addition, collateral circulation can also influence the resultant infarction size and infarction patterns. [5] Currently leptomeningeal anastomoses is considered as an important collateral flow path in M1-MCA occlusion patients. However, collateral flow through other paths, such as small branches of MCA or moyamoya vessels could also be an important factor influencing the progression of patients. [6] The anterior temporal artery (ATA) is the first major branch of MCA. The presence of ATA is often encountered in patients with atherosclerotic MCA occlusion in which it may provide important collateral flow for these patients.

In this study, we hypothesized that presence of ATA associates with good outcome, defined as modified Rankin Scale $(\mathrm{mRS}) \leq 2$ at 3 months post atherosclerotic M1-MCA occlusion.

\section{Patients and Methods}

\section{Study Design and Patient Selection}

All patients in our study gave informed consent for participation. This study was approved by the Institutional Review Board of Jinling Hospital (Nanjing, China) and has therefore been performed in accordance with the ethical standards laid down in the 1964 Declaration of Helsinki and its later amendments. Data for this study was retrieved from the Nanjing Stroke Registry Program (NSRP). [7] From this registry, we included consecutive patients with acute atherosclerotic MCA occlusion between January 2007 and December 2012. All patients were presented with a focal neurological deficit with a duration of $>6$ hours and $<1$ week. Patients suspected of M1-MCA occlusion by TCD, MRA or CTA underwent conventional angiography to definite M1-MCA occlusion diagnosis, evaluate the collateral circulation and guild therapy of these patients. MRI scans were performed to determine the lesion distribution and infarct patterns and conventional angiography data was collected to determine the presence of anterior temporal artery and evaluate the collateral circulation. 
Patients were included in our study if they had (1) a large-artery atherosclerosis stroke diagnosis in accord with TOAST criterion, (2) acute ischemic lesions distributed within MCA territory or border-zone areas with DWI confirmation in 1 week of symptom onset, (3) conventional angiography demonstrating ipsilateral MCA-M1 occlusion in 2 weeks of symptom onset, (4) present with a focal neurological deficit with a duration of $>6$ hours and 1 weeks. We excluded patients who had (1) an age of <18 years old, (2) infarcts in multiple vascular territories beyond the unilateral MCA territory, (3) non-atherosclerotic MCA occlusion (eg, Moyamoya disease, radiation-induced arteriopathy, dissection, cardioembolic or arteritis), (4) concomitant tandem ICA and MCA diseases (eg, ipsilateral ICA stenosis $\geq 50 \%$ ), (5) no MRI scan or conventional angiography, (6)lost follow-up.

We collected baseline characteristics for these participants, including sex, age, body mass index(BMI) as well as vascular risk factors and pre-event medication (eg, antiplatelet, antihypertensive and statin). Admission characteristics, including National Institutes of Health Stroke Scale (NIHSS) score, blood glucose, C-reactive protein (CRP), and systolic blood pressure (SBP), and therapeutic methods after admission were also collected.

At the 3-month follow-up, patients were interviewed in the outpatient clinic or by telephone to evaluate clinical outcome. Good outcome at 3 months was defined as modified Rankin Scale score $\leq 2$.

\section{MRI Infarction Patterns and DWI ASPECTS}

All patients were scanned within one week of symptom onset with 1.5 Tesla or 3 Tesla MRI scanners and a head coil. Acute infarcts on DWI were defined as hyperintensities on the DWI and hypointensities on the apparent diffusion coefficient map.

DWI lesion patterns and DWI ASPECTS were analyzed by two investigators (Yongkun Li and Wen Sun) who were blinded to clinical and angiography data. The topography of ischemic lesions was determined using published templates. [8] Lesion distribution was analyzed according to their infarct location in which MCA vascular structure were divided into the following territories: perforator, pial, and borderzone. Correspondingly, infarcts were named to match their occurring territory: perforator artery infarct (PAI) refers to striatocapsular infarcts or perforating vessel infarcts of the MCA; pial infarct (PI) refers to infarcts occurring in vascular territories supplied by the main leptomeningeal branches of the MCA; 
borderzone infarct (BZI) refers to infarcts occurring in the anterior or posterior cortical BZ or internal BZ of the MCA. In extension, large territorial infarct (LTI) refers to a large ischemic lesion involving the cerebral cortex and subcortical structures in one or more M2 artery territories.

Infarct patterns were divided into two main categories: single territorial lesion and multiple territorial lesions. Therefore, lesions could be categorized to one of the following patterns: single territorial lesions(1)PAI; (2)PI; (3)BZI; (4)LTI; multiple territorial lesions- (5)PAI+PI; (6)PAI+PI+BZ; (7)PAI+BZ; (8) $\mathrm{PI}+\mathrm{BZ}$.

The role of DWI-ASPECTS substituting for lesion volume in acute stroke patients has been well established.[9] To analyze the difference of lesion size on DWI between the two groups, we assessed ASPECTS on DWI according to the method used by Barber et al. [10] ASPECTS includes ten original ASPECTS regions, assigning a score of 1 for a normal and a score of 0 for a region showing ischemic change.

Angiography and Collateral Circulation

To evaluate the collateral circulation and guild therapy of these patients, patients suspected of M1-MCA occlusion by TCD, MRA or CTA underwent conventional angiography within two weeks of symptom onset. We used the Seldinger method via the transfemoral approach and catheterization of the bilateral carotid and vertebral arteries with a 5F selective catheter. MCA occlusion was defined as Thrombolysis in Myocardial Infarction $($ TIMI $)=0$. The presence of ATA and the location of MCA occlusion were also evaluated. Informed consent obtained from all patients before angiography.

Collateral flow was assessed with the American Society of Interventional and Therapeutic Neuroradiology (ASITN)/Society of Interventional Radiology (SIR) Collateral Flow Grading System. Two vascular neurologists (Gelin Xu and Renliang Zhang) who were blinded to the clinical and MRI variables independently analyzed the angiographic data. In the event of discrepancies, the final result was adopted by consensus. ASTIN/SIR score with 3-4 was considered as good collateral circulation.

Statistical Analysis

We compared differences between patients with or without ATA using t test or Mann-Whitney test for 
continuous variables and Chi-aquare test or Fisher exact tests for categorical variables as appropriate. Correlation between DWI-ASPECTS, Distal M1 occlusion, ASTIN/SIR and Presence of ATA was analyzed by calculating Spearman correlation coefficient. To identify independent predictors of favorable outcome, we performed binary logistic regression analysis in which all covariates with a $\mathrm{P}$ value $<0.1$ in a univariate analysis were entered into this logistic regression model. A value of $\mathrm{P}<0.05$ was used to indicate statistical significance.

\section{Results}

\section{Patients and Clinical Characteristics}

In our study, a total of 103 patients met entry criteria with only 5 patients losing follow-up. Baseline characteristics of patients are shown in Table 1. Among them, forty-four $(44 / 98,44.9 \%)$ patients were found to have ATA present while fifty-four $(54 / 98,55.1 \%)$ patients were found to have ATA absent. The anterior temporal artery absent (ATAA) group was found to higher rates of hypertension than the anterior temporal artery present (ATAP) group $(72.2 \%$ vs $52.3 \%, \mathrm{p}=0.042)$. Other risk factors and baseline characteristics (including SBP, CRP and blood glucose) between the two groups were similar. Additionally, the baseline National Institutes of Health Stroke Scale (NIHSS) on admission is significantly higher in the ATAA group than that of the ATAP group $(\mathrm{p}=0.043)$. Furthermore, baseline pre-event medication and medication received during hospitalization between these two groups were similar.

\section{Infarct Characteristics and Collateral Circulation}

To investigate whether the volume of infarcts varies between these two groups, we analyzed patient DWI-ASPECTS through MRI. Data in Table 1 shows that the number of patients with ASPECTS $\geq 7$ of the ATAA group is smaller than that of the ATAP group $(\mathrm{p}=0.034)$. The number of lesions and the distribution of lesions in all patients were also analyzed (Figure 1), in which the presence of multiple lesions were more common in the ATAA group than the ATAP group (81.5\% vs $63.3 \% \mathrm{p}=0.047)$. Pertaining to vascular territorial distribution of infarcts, PAI were similar between the two groups, however, small perforating artery infarcts $(<2 \mathrm{~cm})$ occurred more frequently in patients with ATA present than those without $(22.7 \%$ vs $5.6 \% \mathrm{p}=0.013)$; large perforating artery infarcts $(\geq 2 \mathrm{~cm})$ were more common 
in the ATAA group (44.4\% vs $25.0 \% \mathrm{p}=0.046)$ whereas PI and BZI occurrence showed minimal

differences between the two groups.

With regard to multiple territorial lesions, the most frequently occurring lesion pattern in the ATAA group is the concomitant PAI and BZ infarct (10/54, 18.5\%)while the ATAP group varied in occurrence: 2 $(4.5 \%), 6(13.6 \%), 3(6.8 \%), 6(13.6 \%)$ patients had PAI+PI, PAI+PI+BZI, PAI+BZI, PI+BZI respectively. There was no difference in collateral flow between the two groups (Table 1).

Good Clinical Outcome at 3 Months

At 3 months, 50/98 patients (51.0\%) had a good clinical outcome. As shown in Table 2, the good outcome group had a lower baseline NIHSS score, higher prevalence rate of ATA, more patients with ASPECTS $\geq 7$, and more single BZ territory lesions on DWI than the group with bad outcome. Hence, the presence of ATA (OR, 4.45; 95\% CI, 1.52 to $13.03 ; \mathrm{P}=0.007)$ is independently associated with good outcome (Table 2). As shown in Table 3, presence of ATA correlated well with high DWI-ASPECTS and distal M1 occlusion.

\section{Discussion}

Our study found that in acute atherosclerotic M1-MCA occlusion, patients with ATA present have smaller lesion volume, a higher number of small perforating artery infarcts, better baseline neurological function, and less multiple lesion occurrence rates. Hence, the presence of ATA was determined to be independently associated with good clinical outcomes.

Intracranial atherosclerosis disease is the most common cause of ischemic stroke in China.[11] In patients with intracranial atherosclerosis artery occlusion, collateral flow plays an important role in maintaining downstream territory perfusion. For M1-MCA occlusion, primary collateral circulation is established via the leptomeningeal anastomoses (LMAs) from the anterior cerebral artery (ACA) and posterior cerebral artery (PCA). In previous studies, the presence of ATA in M1-MCA occlusion on CTA identifies a group of patients with reduced case fatality.[12] Recent study [13] also assessed leptomeningeal collateral status with whole-brain dynamic time-resolved computed tomography angiography (CTA). Unlike a conventional angiography, this technique visualizes pial arterial filling in all vascular territories, thereby 
providing additional hemodynamic information. It might be better at estimating collateral status. Our study included patients between January 2007 and December 2012, we couldn't analysis collateral status with whole-brain dynamic time-resolved computed tomography angiography in the last few years. Then, conventional digital subtraction angiography (DSA) was performed to evaluate collateral circulation and guild therapy of these patients. Although we did ICA injections to analysis ACA-MCA collateras and vertebral injection to measure PCA-MCA collaterals, we couldn't estimate these collateral status simultaneously like whole-brain dynamic time-resolved CTA. So, further studies about collateral circulation could be based on whole-brain dynamic time-resolved CTA. Our results showed that our patients have similar collateral circulation from ACA, PCA, and LMA, which indicates that there may be other mechanisms influencing patient outcome.

Before undergoing conventional DSA, patients in our study also underwent MRI and DWI to evaluate lesion size and infarct pattern. DWI-ASPECTS is a semi-quantitative estimation of lesion size, in which it has been shown to correlate well with actual DWI lesion volume. [9,14] Lesion size on DWI has been demonstrated to be associated with stroke severity and has also been studied as an important predictor of functional outcome. $[14,15]$ We analyzed DWI-ASPECTS to investigate the difference of lesion volume between the two groups. From our results we found that the presence of ATA was associated with higher DWI-ASPECTS. As we know, ATA is the first major branch of the MCA, it provides blood supply to the polar and anterolateral portions of the temporal lobe [16] .Therefore, the presence of ATA in M1-MCA occlusions may negate proximal occlusion of the MCA trunk, which has been identified previously as an angiographic factor predicting malignant MCA infarction.

Lesion pattern is another factor affecting the association between ATA presence and outcome. Previous studies suggested that advanced injury in the basal ganglia resulted in worse dysfunction and disability at discharge among patients with M1-MCA occlusion. [17-19] Our results show that PAI occurrence is smaller in the ATAP group than the ATAA group. This can be accounted by the difference in M1-MCA occlusion position, in which the ATA commonly arises proximal to or opposite to the origin of the lenticulostriate arteries. In acute atherosclerosis M1-MCA occlusion patients, ATA presence suggests that only the smaller perforator artery territory is involved[20] . Furthermore, ATA presence can also sustain downstream perfusion from MCA occlusions, involving smaller downstream MCA territory. 
A combination of local branch occlusion and embolism, with or without hemodynamic compromise, is a common pathogenesis of stroke resulting from MCA disease[21]. Our data also provides evidence that multiple DWI lesions, which may be markers of embolism, are more common in the ATAA group. These lesions on DWI may be caused by an embolism originating from the proximal MCA lodging itself in the distal MCA. Additionally, perfusion deficits caused by MCA occlusion may contribute to the development of embolic infarction through impaired clearance of emboli. In ATAP patients, ATA can partially maintain perfusion, leading to enhanced clearance of emboli in the MCA territory.

There are two main strengths in our study: the first being that we have only included patients with acute atherosclerotic MCA infarction, allowing us to maintain the consistency of our patients. The second being that all our patients have underwent both conventional DSA and MRI scan. Conventional DSA is considered to be the "gold standard" for determination of ATA presence as well as collateral flow evaluation while DWI is the most sensitive diagnostic modality for detecting acute infarction and lesion pattern[21]. Therefore, these methods allow us to better explore the potential mechanisms underlying the relationship between ATA presence and good outcome.

While we found that patients with ATA generally had better anterior flow than ATAA group patients, we did not have CT-Perfusion or MRI-Perfusion results to confirm the link between ATA presence and better perfusion Therefore in the future studies, the association between ATA presence and downstream perfusion areas from MCA occlusion site worthy of further investigation. Additionally, although we collected patients in a prospective stroke registry, this study was a single-center study and was limited by a small sample size. 


\section{Ethical Standards and Patient Consent}

We declare that this study has been approved by the Institutional Review Board of Jinling Hospital (Nanjing, China) and has therefore been performed in accordance with the ethical standards laid down in the 1964 Declaration of Helsinki and its later amendments. We declare that all patients gave informed consent prior to inclusion in this study.

\section{Acknowledgements}

This study was supported by grants from National Natural Science Foundation of China (Grant no. 81000501), Jinling Hospital Medical Scientific Research Projects (2012003), and the Clinical Medical Center Program of Jiangsu Province (Neurology Center). The funders had no role in study design, data collection and analysis, decision to publish, or preparation of the manuscript. We thank Nathan Xuan Cong for help with revising this manuscript.

\section{Conflict of interest}

We declare that we have no conflict of interest. 


\section{References}

1. Kim YS, Garami Z, Mikulik R, Molina CA, Alexandrov AV (2005) Early recanalization rates and clinical outcomes in patients with tandem internal carotid artery/middle cerebral artery occlusion and isolated middle cerebral artery occlusion. Stroke; a journal of cerebral circulation 36 (4):869-871.

doi:10.1161/01.STR.0000160007.57787.4c

2. Tsai NW, Chang HW, Chang WN, Huang CR, Lin TK, Chen SD, Lui CC, Wang KW, Cheng BC, Hung PL, Chang CS, Lu CH (2005) Prognostic factors and therapeutic outcome of isolated symptomatic middle cerebral artery stenosis. European journal of neurology : the official journal of the European Federation of Neurological Societies 12 (7):519-526. doi:10.1111/j.1468-1331.2005.00995.x

3. Nuutinen J, Liu Y, Laakso MP, Karonen JO, Roivainen R, Vanninen RL, Partanen K, Ostergaard L, Sivenius $\mathrm{J}$, Aronen $\mathrm{HJ}$ (2006) Assessing the outcome of stroke: a comparison between MRI and clinical stroke scales. Acta neurologica Scandinavica 113 (2):100-107. doi:10.1111/j.1600-0404.2005.00550.x

4. Calleja Al, Cortijo E, Garcia-Bermejo P, Gomez RD, Perez-Fernandez S, Del Monte JM, Munoz MF, Fernandez-Herranz R, Arenillas JF (2013) Collateral circulation on perfusion-computed

tomography-source images predicts the response to stroke intravenous thrombolysis. European journal of neurology : the official journal of the European Federation of Neurological Societies 20 (5):795-802. doi:10.1111/ene.12063

5. Liebeskind DS, Cotsonis GA, Saver JL, Lynn MJ, Turan TN, Cloft HJ, Chimowitz MI, Investigators W (2011) Collaterals Dramatically Alter Stroke Risk in Intracranial Atherosclerosis. Ann Neurol 69 (6):963-974. doi:10.1002/ana.22354

6. Frolich AM, Psychogios MN, Klotz E, Schramm R, Knauth M, Schramm P (2012) Antegrade flow across incomplete vessel occlusions can be distinguished from retrograde collateral flow using 4-dimensional computed tomographic angiography. Stroke; a journal of cerebral circulation 43 (11):2974-2979. doi:10.1161/strokeaha.112.668889

7. Liu X, Xu G, Wu W, Zhang R, Yin Q, Zhu W (2006) Subtypes and one-year survival of first-ever stroke in Chinese patients: The Nanjing Stroke Registry. Cerebrovascular diseases (Basel, Switzerland) 22 (2-3):130-136. doi:10.1159/000093241

8. Tatu L, Moulin T, Bogousslavsky J, Duvernoy H (1998) Arterial territories of the human brain: cerebral hemispheres. Neurology 50 (6):1699-1708

9. de Margerie-Mellon C, Turc G, Tisserand M, Naggara O, Calvet D, Legrand L, Meder JF, Mas JL, Baron JC, Oppenheim C (2013) Can DWI-ASPECTS Substitute for Lesion Volume in Acute Stroke? Stroke; a journal of cerebral circulation. doi:10.1161/strokeaha.113.003047

10. Barber PA, Demchuk AM, Zhang J, Buchan AM (2000) Validity and reliability of a quantitative computed tomography score in predicting outcome of hyperacute stroke before thrombolytic therapy. ASPECTS Study Group. Alberta Stroke Programme Early CT Score. Lancet 355 (9216):1670-1674 11. Arenillas JF (2011) Intracranial atherosclerosis: current concepts. Stroke; a journal of cerebral circulation 42 (1 Suppl):S20-23. doi:10.1161/strokeaha.110.597278

12. Menon BK, Bal S, Modi J, Sohn SI, Watson TW, Hill MD, Demchuk AM, Goyal M (2012) Anterior temporal artery sign in CT angiography predicts reduced fatal brain edema and mortality in acute M1 middle cerebral artery occlusions. Journal of neuroimaging : official journal of the American Society of Neuroimaging 22 (2):145-148. doi:10.1111/j.1552-6569.2010.00566.x

13. Menon BK, O'Brien B, Bivard A, Spratt NJ, Demchuk AM, Miteff F, Lu X, Levi C, Parsons MW (2013) Assessment of leptomeningeal collaterals using dynamic CT angiography in patients with acute ischemic stroke. Journal of cerebral blood flow and metabolism : official journal of the International Society of Cerebral Blood Flow and Metabolism 33 (3):365-371. doi:10.1038/jcbfm.2012.171

14. Tei H, Uchiyama S, Usui T, Ohara K (2011) Diffusion-weighted ASPECTS as an independent marker for predicting functional outcome. Journal of neurology 258 (4):559-565. doi:10.1007/s00415-010-5787-x 15. Thijs VN, Lansberg MG, Beaulieu C, Marks MP, Moseley ME, Albers GW (2000) Is early ischemic lesion volume on diffusion-weighted imaging an independent predictor of stroke outcome? A multivariable analysis. Stroke; a journal of cerebral circulation 31 (11):2597-2602

16. Umansky F, Dujovny M, Ausman JI, Diaz FG, Mirchandani HG (1988) Anomalies and variations of the middle cerebral artery: a microanatomical study. Neurosurgery 22 (6):1023-1027

17. Rosso C, Colliot O, Valabregue R, Crozier S, Dormont D, Lehericy S, Samson Y (2011) Tissue at risk in 
the deep middle cerebral artery territory is critical to stroke outcome. Neuroradiology 53 (10):763-771. doi:10.1007/s00234-011-0916-5

18. Seitz RJ, Sondermann V, Wittsack HJ, Siebler M (2009) Lesion patterns in successful and failed thrombolysis in middle cerebral artery stroke. Neuroradiology 51 (12):865-871.

doi:10.1007/s00234-009-0576-x

19. Loh Y, Towfighi A, Liebeskind DS, MacArthur DL, Vespa P, Gonzalez NR, Tateshima S, Starkman S, Saver JL, Shi ZS, Jahan R, Vinuela F, Duckwiler GR (2009) Basal ganglionic infarction before mechanical thrombectomy predicts poor outcome. Stroke; a journal of cerebral circulation 40 (10):3315-3320. doi:10.1161/strokeaha.109.551705

20. Thomalla GJ, Kucinski T, Schoder V, Fiehler J, Knab R, Zeumer H, Weiller C, Rother J (2003) Prediction of malignant middle cerebral artery infarction by early perfusion- and diffusion-weighted magnetic resonance imaging. Stroke; a journal of cerebral circulation 34 (8):1892-1899.

doi:10.1161/01.str.0000081985.44625.b6

21. Lee DK, Kim JS, Kwon SU, Yoo SH, Kang DW (2005) Lesion patterns and stroke mechanism in atherosclerotic middle cerebral artery disease: early diffusion-weighted imaging study. Stroke; a journal of cerebral circulation 36 (12):2583-2588. doi:10.1161/01.str.0000189999.19948.14 


\section{Figure legends}

Fig 1 Lesion patterns and angiography characteristics of patients in different groups

A, A 63-year-old man presented with right hypesthesia 2 days before MRI study. DWI showing acute left BZ infarction.

B, Angiography of Figure 1-A showing left M1-MCA occlusion and presence of ATA.

C, A 47-year-old man had sudden onset of aphasia and right hemiplegia 4 days before MRI scan. DWI showing acute large territory infarction of left MCA.

D, Angiography of Figure 1-C showing left M1-MCA occlusion and ATA is absent. 
Table1 Baseline characteristics of patients with or without anterior temporal artery

\begin{tabular}{|c|c|c|c|}
\hline Characteristics & $\operatorname{ATAA}(n=54)$ & $\operatorname{ATAP}(n=44)$ & $p$ Value \\
\hline $\operatorname{Age}($ mean \pm SD $)$ & $58.70 \pm 11.70$ & $59.86 \pm 9.16$ & 0.593 \\
\hline Male n(\%) & $39(72.2)$ & $33(75.0)$ & 0.757 \\
\hline \multicolumn{4}{|l|}{ Risk factors } \\
\hline Hypertension $\mathrm{n}(\%)$ & $39(72.2)$ & $23(52.3)$ & $0.042 *$ \\
\hline Diabetes n(\%) & $13(24.1)$ & $12(27.3)$ & 0.718 \\
\hline Hyperlipidemia n(\%) & $15(27.8)$ & $6(13.6)$ & 0.090 \\
\hline Ever smoker n(\%) & $24(44.4)$ & $22(50.0)$ & 0.584 \\
\hline Alcohol consumption $\mathrm{n}(\%)$ & $16(29.6)$ & $10(22.7)$ & 0.441 \\
\hline Previous stroke/TIA n(\%) & $10(18.5)$ & $6(13.6)$ & 0.515 \\
\hline CAD n $(\%)$ & $7(13.0)$ & $4(9.1)$ & 0.546 \\
\hline Baseline NIHSS score(median, IQR) & $8.5(6.0-13.0)$ & $7.0(4.0-10.0)$ & $0.043^{*}$ \\
\hline BMI & $24.64(22.79-25.75)$ & $24.01(22.58-25.27)$ & 0.287 \\
\hline SBP mmHg(median, IQR) & $141(130.0-148.50)$ & $136.5(125.0-153.75)$ & 0.247 \\
\hline
\end{tabular}


Blood glucose, mg/dl(median, IQR)

CRP(median, IQR)

Pre-event medication $\mathrm{n}(\%)$

Antiplatelet therapy at onset

Statin therapy at onset

Antihypertensive therapy at onset

Medication therapy in hospital n(\%)

Double antiplatelet therapy

Statin therapy

Antihypertensive therapy

Distal M1 occlusion

Good collateral flow on DSA n $(\%)$

ASITN-SIR $=0$

ASITN-SIR=1

ASITN-SIR=2
98.1(87.75-109.8)

$2.95(1.08-5.20)$

19(35.2)

$15(27.8)$

23(42.6)

$45(83.3)$

53(98.1)

$37(68.5)$

11(20.4)

$7(13.0)$

$5(9.3)$

21(38.9)

21(38.9)
$95.40(81.00-126.00)$

0.614

$1.95(1.15-5.60)$

0.702

0.402

0.265

0.390

15(34.1)

32(72.7)

0.203

41(93.2)

0.217

22(50.0)

0.062

$<0.001$

25(56.8)

0.810

2(4.5)

0.454

13(29.5)

0.334

24(54.5) 


\begin{tabular}{|c|c|c|c|}
\hline ASITN-SIR=3 & $4(7.4)$ & $2(4.5)$ & 0.688 \\
\hline ASITN-SIR=4 & $3(5.6)$ & $3(6.8)$ & 1.000 \\
\hline ASPECTS $\geq 7$ on DWI n(\%) & $24(44.4)$ & $29(65.9)$ & $0.034 *$ \\
\hline Multiple infarcts on DWI n(\%) & $44(81.5)$ & $28(63.6)$ & $0.047 *$ \\
\hline \multicolumn{4}{|l|}{ Infarct involving perforating artery territory $\mathrm{n}(\%)$} \\
\hline $\operatorname{Small}(<2 \mathrm{~cm})$ & $3(5.6)$ & $10(22.7)$ & $0.013 *$ \\
\hline $\operatorname{Large}(\geq 2 \mathrm{~cm})$ & $24(44.4)$ & $11(25.0)$ & $0.046^{*}$ \\
\hline Infarct involving pial artery territory $\mathrm{n}(\%)$ & $26(48.1)$ & 18(40.9) & 0.474 \\
\hline Infarct involving Border-zone territory $\mathrm{n}(\%)$ & $32(59.3)$ & $21(47.7)$ & 0.255 \\
\hline \multicolumn{4}{|l|}{ Single territorial lesion $\mathrm{n}(\%)$} \\
\hline 1.PAI & $7(13.0)$ & $10(22.7)$ & 0.204 \\
\hline 2.PI & $9(16.7)$ & $4(9.1)$ & 0.271 \\
\hline 3.BZI & $8(14.8)$ & $6(13.6)$ & 0.868 \\
\hline 4.Large territorial & $3(5.6)$ & $7(15.9)$ & 0.092 \\
\hline
\end{tabular}

Multiple territorial lesions n(\%) 


\begin{tabular}{llcc}
$5 . \mathrm{PAI}+\mathrm{PI}$ & $3(5.6)$ & $2(4.5)$ & 1.000 \\
6.PAI+PI+BZI & $7(13.0)$ & $6(13.6)$ & 0.922 \\
$7 . \mathrm{PAI}+\mathrm{BZI}$ & $10(18.5)$ & $3(6.8)$ & 0.089 \\
$8 . \mathrm{PI}+\mathrm{BZI}$ & $7(13.0)$ & $6(13.6)$ & 0.922 \\
\hline
\end{tabular}

\section{$* P<0.05$}

Abbreviations: $\mathrm{TIA}=$ transient ischemic attack, $\mathrm{CAD}=$ coronary artery disease, $\mathrm{IQR}=$ inter quartile range, $\mathrm{BMI}=$ body $\operatorname{mass}$ index, $\mathrm{SBP}=$ systolic blood pressure, $\mathrm{CRP}=\mathrm{C}$-reactive protein, $\mathrm{DSA}=$ digital subtraction angiography, $\mathrm{DWI}=$ diffusion weighted imaging, $\mathrm{PAI}=$ perforator artery infarct, $\mathrm{PI}=$ pial infarct, $\mathrm{BZI}=$ borderzone infarct 
Table2 Factors associated with good clinical outcome

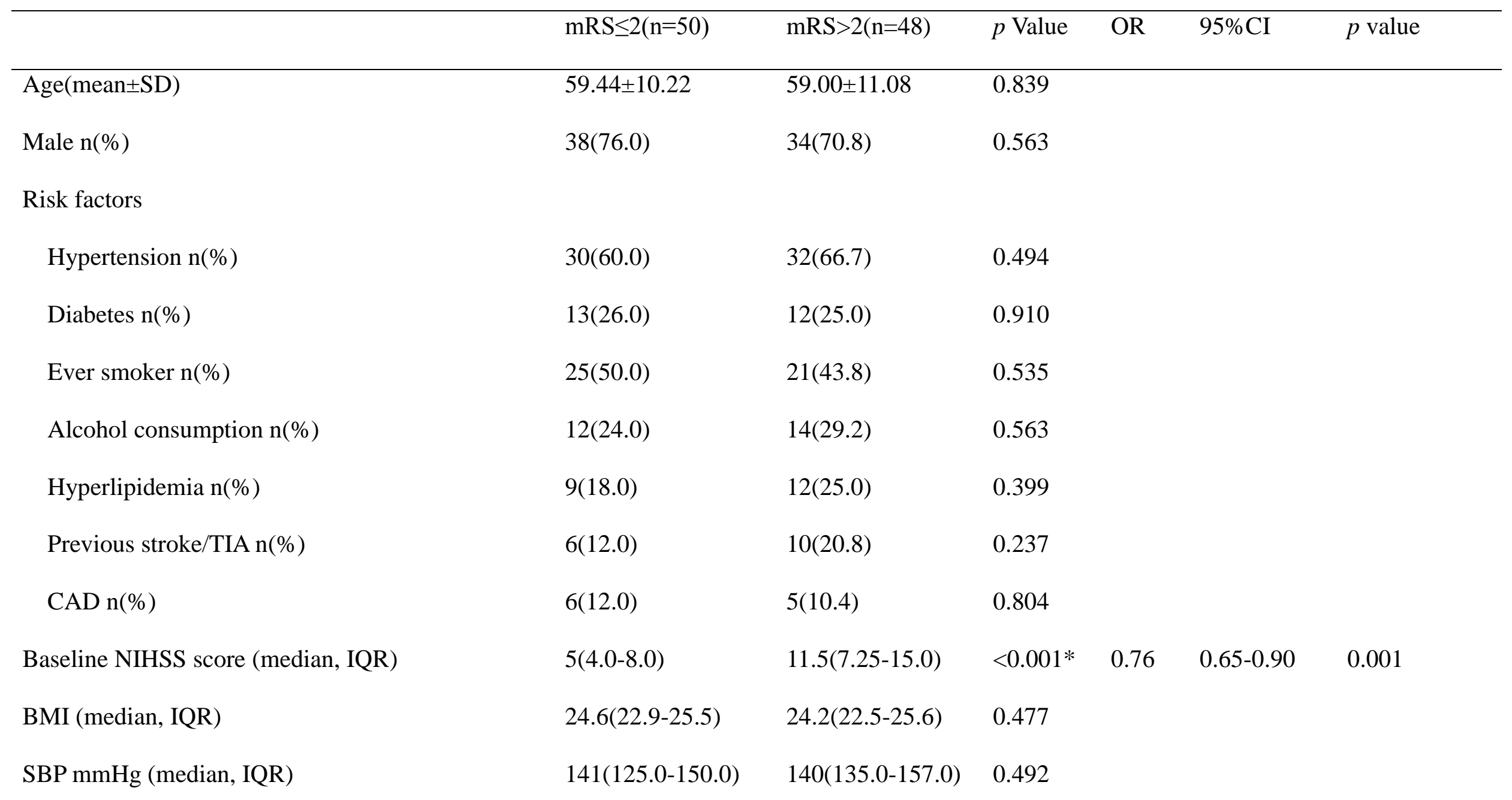


Blood glucose, mg/dl (median, IQR)

CRP (median, IQR)

Pre-event medication

Antiplatelet therapy at onset $\mathrm{n}(\%)$

Statin therapy at onset $\mathrm{n}(\%)$

Antihypertensive therapy at onset $\mathrm{n}(\%)$

Medication therapy in hospital

Double antiplatelet therapy $\mathrm{n}(\%)$

Statin therapy $n(\%)$

Antihypertensive therapy $\mathrm{n}(\%)$

Presence of ATA

Distal M1 occlusion

Good retrograde collateral flow on DSA n(\%)

ASITN-SIR $=0$

ASITN-SIR $=1$
$95.4(82.35-109.80) \quad 98.1(82.8-125.1) \quad 0.391$

$1.90(0.98-3.93) \quad 3.00(1.40-7.28) \quad 0.110$

$\begin{array}{lll}13(26.0) & 18(37.5) & 0.221 \\ 11(22.0) & 12(25.0) & 0.726 \\ 20(40.0) & 18(37.5) & 0.800\end{array}$

$\begin{array}{lll}37(74.0) & 40(83.3) & 0.260\end{array}$

$\begin{array}{lll}47(94.0) & 47(97.9) & 0.327\end{array}$

$\begin{array}{lll}29(58.0) & 30(62.5) & 0.649\end{array}$

$\begin{array}{lll}31(62.0) & 13(27.1) & 0.001 *\end{array}$

$4.45 \quad 1.52-13.03 \quad 0.007$

$22(44.0)$

14(29.2)

0.128

5(10.0)

7(14.6)

0.489

5(10.0)

2(4.2)

0.436

17(34.0)

17(35.4)

0.883 


\begin{tabular}{|c|c|c|c|c|c|c|}
\hline ASITN-SIR=2 & $23(46.0)$ & $22(45.8)$ & 0.987 & & & \\
\hline ASITN-SIR=3 & $1(2.0)$ & $5(10.4)$ & 0.108 & & & \\
\hline ASITN-SIR=4 & $4(8.0)$ & $2(4.2)$ & 0.678 & & & \\
\hline ASPECTS $\geq 7$ on DWI $n(\%)$ & $39(78.0)$ & $14(29.2)$ & $<0.001^{*}$ & 2.65 & $0.87-8.07$ & 0.086 \\
\hline Multiple infarcts on DWI n (\%) & $34(68.0)$ & $38(79.2)$ & 0.211 & & & \\
\hline \multicolumn{7}{|l|}{ Infarct involving perforating artery territory $\mathrm{n}(\%)$} \\
\hline $\operatorname{Small}(<2 \mathrm{~cm})$ & $9(18.0)$ & $4(8.3)$ & 0.158 & & & \\
\hline Large $(\geq 2 \mathrm{~cm})$ & $15(30.0)$ & $20(41.7)$ & 0.228 & & & \\
\hline Infarct involving pial artery territory $\mathrm{n}(\%)$ & $21(42.0)$ & $23(47.9)$ & 0.556 & & & \\
\hline Infarct involving Border-zone territory $\mathrm{n}(\%)$ & $23(46.0)$ & $30(62.5)$ & 0.101 & & & \\
\hline \multicolumn{7}{|l|}{ Single territorial lesion } \\
\hline PAI territorial lesion $\mathrm{n}(\%)$ & $11(22.0)$ & $6(12.5)$ & 0.214 & & & \\
\hline PI territorial lesion $\mathrm{n}(\%)$ & $9(18.0)$ & $4(8.3)$ & 0.158 & & & \\
\hline $\mathrm{BZ}$ territorial lesion $\mathrm{n}(\%)$ & $10(20.0)$ & $4(8.3)$ & $0.099 *$ & 1.25 & $0.30-5.25$ & 0.761 \\
\hline Large territorial lesion $\mathrm{n}(\%)$ & $3(6.0)$ & $7(14.6)$ & 0.161 & & & \\
\hline
\end{tabular}


Multiple territorial lesions

$\begin{array}{lccr}\text { PAI+PI territorial lesion n (\%) } & 4(8.0) & 1(2.1) & 0.362 \\ \text { PAI+PI+BZ territorial lesion n (\%) } & 4(8.0) & 9(18.8) & 0.117 \\ \text { PAI+BZ territorial lesion n (\%) } & 5(10.0) & 8(16.7) & 0.331 \\ \text { PI+BZ territorial lesion n (\%) } & 4(8.0) & 9(18.8) & 0.117\end{array}$

$* P<0.1$

Abbreviations: $\mathrm{TIA}=$ transient ischemic attack, $\mathrm{CAD}=$ coronary artery disease, $\mathrm{IQR}=$ inter quartile range, $\mathrm{BMI}=$ body mass index, $\mathrm{SBP}=$

systolic blood pressure, $\mathrm{CRP}=\mathrm{C}$-reactive protein, $\mathrm{DSA}=$ digital subtraction angiography, $\mathrm{DWI}=$ diffusion weighted imaging, $\mathrm{PAI}=$

perforator artery infarct, $\mathrm{PI}=$ pial infarct, $\mathrm{BZ}=$ borderzone infarct 
Table 3 Spearman correlation analysis between DWI-ASPECTS, Distal M1 occlusion, ASTIN/SIR and Presence of ATA

\begin{tabular}{clll}
\hline & DWI-ASPECTS & Distal M1 occlusion & ASITN/SIR \\
\hline Presence of ATA & & & \\
$\mathrm{R}$ & $0.223^{*}$ & $0.376^{*}$ & 0.117 \\
$\mathrm{p}$ & 0.027 & 0.000 & 0.250 \\
$\mathrm{~N}$ & 98 & 98 & 98 \\
\hline$* P<0.05$ & &
\end{tabular}

$* P<0.05$ 


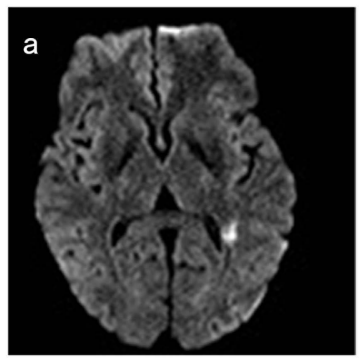

b
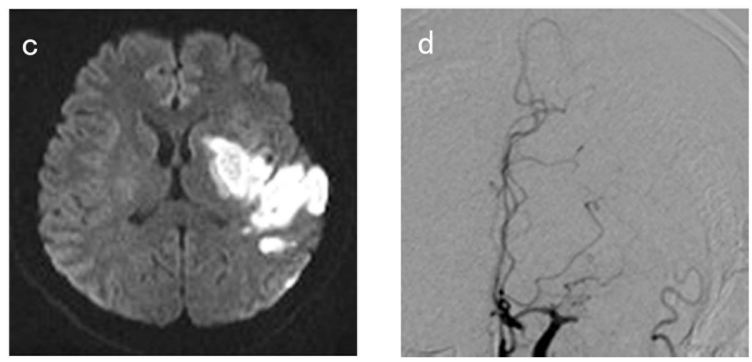